\title{
Numerical Simulation of Lasing Dynamics in Choresteric Liquid Crystal Based on ADE-FDTD Method
}

\author{
Tatsunosuke Matsui \\ Mie University \\ Japan
}

\section{Introduction}

Liquid crystals (LCs) are categorized in one class of condensed materials which show both character of liquids and solids (crystals). Liquid-like fluidic character of LCs allows them to show dynamic response to external stimuli such as electrical, optical and magnetic fields. Anisotropic characters of LCs like crystals show determines the way how they respond to external stimuli and also how they appear (de Gennes \& Prost, 1995). These characteristics are widely utilized in LC display devices. LCs are further categorized in subgroups (phases) in terms of their degrees of order (orientational and positional) as shown in Fig. 1.

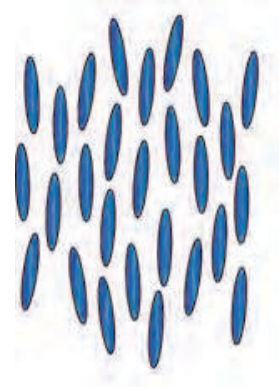

nematic

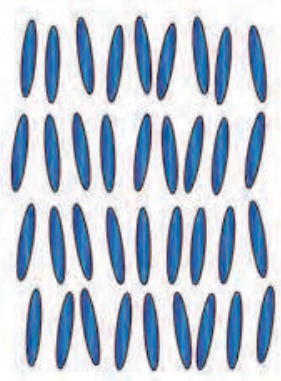

smectic A

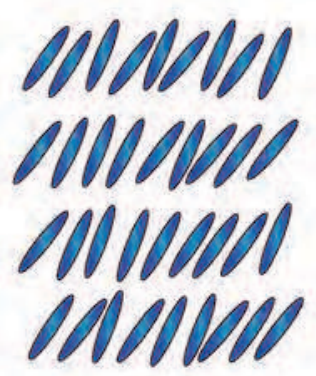

smectic C

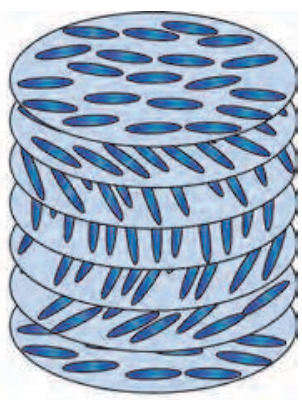

cholesteric

Fig. 1. Schematics of several liquid crystal phases.

Cholesteric liquid crystal (CLC) is one of these sub-phases of LCs and the self-organized formation of periodic helical structure is the most significant characters of this phase from the viewpoint of device application. In case the pitch of the helix of CLCs is in the range of the wavelength of visible light, they selectively reflect part of incident light in a certain manner determined by their refractive index and the sense of helix. Figure 2 shows the simulated transmission and reflection spectra of right-handed-circularly-polarized light normally incident on CLC. Transmission/reflection band (stop band) can be recognized. The central frequency and the bandwidth of the band are dependent on both refractive indices and pitch of helix of CLC. 


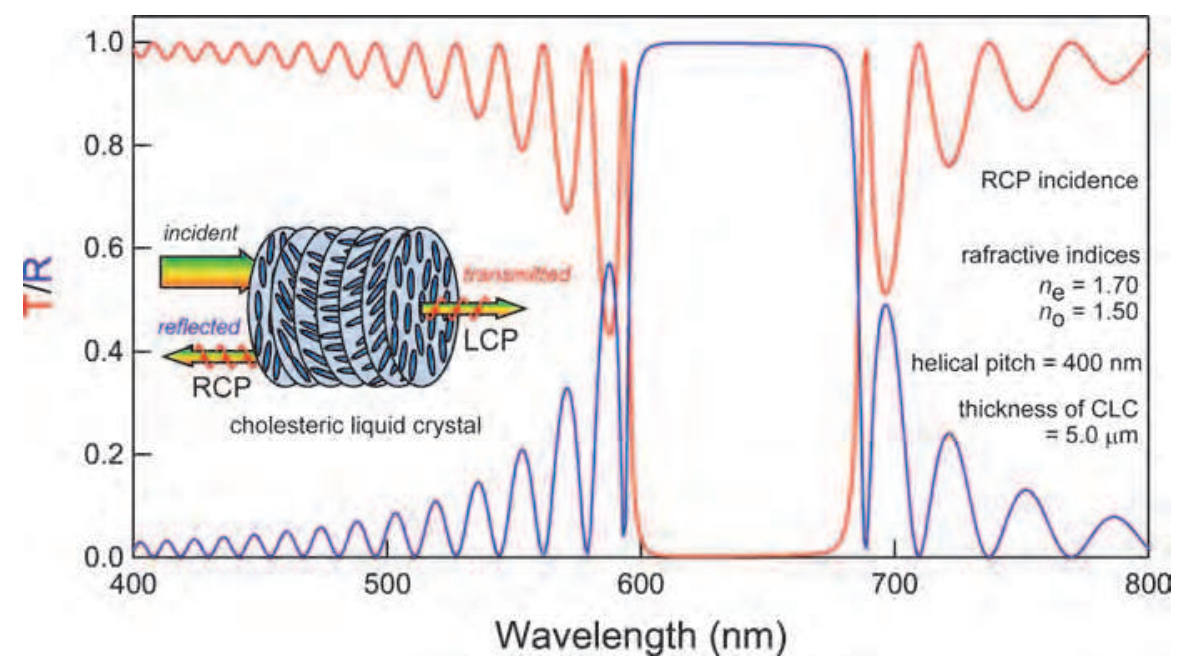

Fig. 2. Transmission and reflection spectra simulated by Berreman's $4 \times 4$ transfer matrix method. Right-handed circularly polarized light is supposed to normally incident on $5 \mu \mathrm{m}-$ thick CLC with $400 \mathrm{~nm}$-pitch right-handed helix. Extraordinary and ordinary refractive indices are 1.70 and 1.50, respectively. (Inset) schematic representation of selective reflection by CLC with right-handed helix. As an incident light, linearly or randomly polarized and in the photonic band wavelength is assumed.

So many studies have been made to utilize this so-called "selective reflection" character of CLC to make the reflection type display, or in other words, electronic paper. Recently, CLCs are also extensively studied as a photonic band gap (PBG) material or photonic crystal (PC). In 1987, Yablonovitch (Yablonovitch, 1987) and John (John, 1987) put forward the basic concept of PBG and since then so many studies have been carried out. Electromagnetic (EM) wave (photons) propagating in PCs composed of periodic stacking of dielectric materials with different dielectric permittivity behaves just like de Broglie wave (electrons) travelling in periodic Coulomb potential in crystals. Long dwell time of photon at PBG edge energy allows strong light-matter interaction and low threshold lasing may be obtained in such PBG system with optical gain introduced (Dowling et al, 1994). Introducing defect states in PC can induce photon localization in PC (Joannopoulos et al., 1995).

Periodic helical structure of CLC can also work as PBG material. In 1998, Kopp and his coworkers succeeded in obtaining band-edge lasing from dye-doped CLC (Kopp et al., 1998). Since their pioneering work, numerous studies have been carried out from the viewpoint of academic interest and technical applications (Coles \& Morris, 2010). The introduction of various types of defects has also been attempted in CLC as schematically summarized in Fig. 3. Yang and his coworkers showed, based on numerical analysis, that introduction of isotropic thin layer as a defect in the middle of CLC layer (Fig. 3 (a)) creates the defect state (narrow transmission band) in the stop band, which can be tuned via altering thickness or refractive index of the defect layer (Yang et al., 1999). Kopp and Genack have numerically demonstrated that twist-defect, discontinuous phase shift of the helical twist of CLC molecules (Fig. 3 (b)), could indeed function as a defect (Kopp \& Genack, 2002). This type of defect is unique in CLC with optical anisotropy. Lasing from twist-defect has 
been experimentally attained utilizing photopolymerized CLC polymer films (Ozaki et al., 2003; Schmidtke et al., 2003). It has been also shown that introducing defect can contribute to reduce lasing threshold. Other types of defects have also been introduced. It has been numerically shown that local modulation of helical pitch of CLC as schematically shown in Fig. 3 (c) can also introduce defect states in the stop band (Matsui et al., 2004). Multi-layer of CLCs with different helical pitch as shown in Fig. 3 (d) has been experimentally realized, which have been successful to achieve reduced lasing threshold (Ozaki et al., 2006; Takanishi et al., 2007).

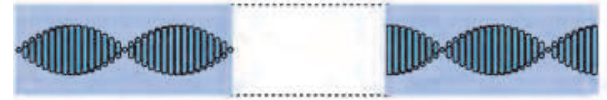

(a) Isotropic defect

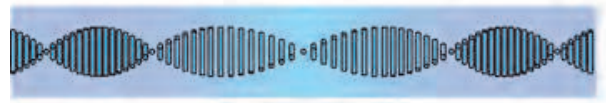

(c) gradient pitch

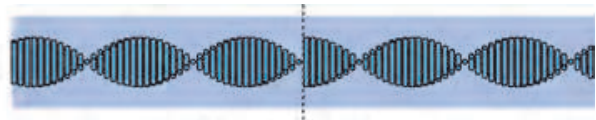

(b) twist defect

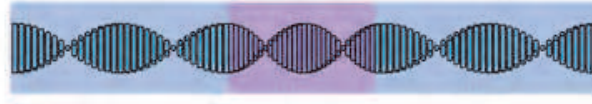

(d) multi-layer

Fig. 3. Schematic representations of proposed defects in CLC PCs. (a) isotropic defect (b) twist defect (c) locally modulated pitch (d) multi-layer system.

Refining device architecture may realize further lowering of the lasing threshold, which motivated us to develop numerical simulation technique for the development of the more efficient laser device architectures. We have recently reported on numerical simulation of lasing dynamics in CLCs (Matsui \& Kitaguchi, 2010). We have employed an auxiliary differential equation finite-difference time-domain (ADE-FDTD) method, which was first applied to the analysis of random lasing in one-dimensional (1D) random system (Jiang \& Soukoulis, 2000). We have successfully reproduced circularly-polarized lasing in CLC at the energy of the edge of the stop band. Moreover, as will be discussed later, we have also shown that our computational scheme can also be utilized to search for more efficient device architecture with reduced lasing threshold. Here we will summarize the computational procedure of ADE-FDTD method for the analysis of lasing dynamics in CLC and show that this technique is quite useful for the analysis of EM field dynamics in and out of CLC laser cavity under lasing condition, which might contribute to the deep understanding of the underlying physical mechanism of lasing dynamics in CLC.

\section{Numerical simulations}

In this section, numerical simulation techniques employed in this study (1) ADE-FDTD method for the analysis of lasing dynamics and (2) Berreman's $4 \times 4$ transfer matrix method for the analysis of transmission and reflection spectra are summarized. As will be discussed, ADEFDTD approach enables us to analyze lasing dynamics in CLC from various viewpoints such as time-dependent EM fields, Fourier-transformed emission spectra and snapshots of spatialdistributions of EM fields. Berreman's $4 \times 4$ transfer matrix method is traditionally employed to simulate transmission and reflection spectra in rather simpler way. 


\subsection{ADE-FDTD based numerical simulation of lasing dynamics}

In this subsection, overview of ADE-FDTD approaches for the analysis of lasing dynamics in various types of micro- and nano-laser systems made so far will be given first, and then detailed numerical procedure will be given.

\subsubsection{Overview of ADE-FDTD approach for the analysis of lasing dynamics in micro- and nano-photonics systems}

FDTD has been widely utilized to numerically simulate the propagation and/or localization of EM waves in micro- and nano-photonic media (Taflove \& Hagness, 2005). In order to investigate lasing dynamics, ADE-FDTD approaches have also been developed, in which the FDTD method is usually coupled with the rate equation in a four-level energy structure and the equation of motion of polarization (Nagra \& York, 1998) as schematically shown in Fig. 4. As discussed above, Jiang and Soukoulis have employed ADE-FDTD method for the analysis of random lasing in 1D random system (Jiang \& Soukoulis, 2000). Numerous groups have followed them to investigate lasing dynamics in various kinds of laser cavities such as 2D random media (Vanneste \& Sebbah, 2001), PCs (Bermel et al., 2006; Shi \& Prather, 2007), and distributed Bragg reflectors (Chang \& Taflove, 2004; Redding et al., 2008).

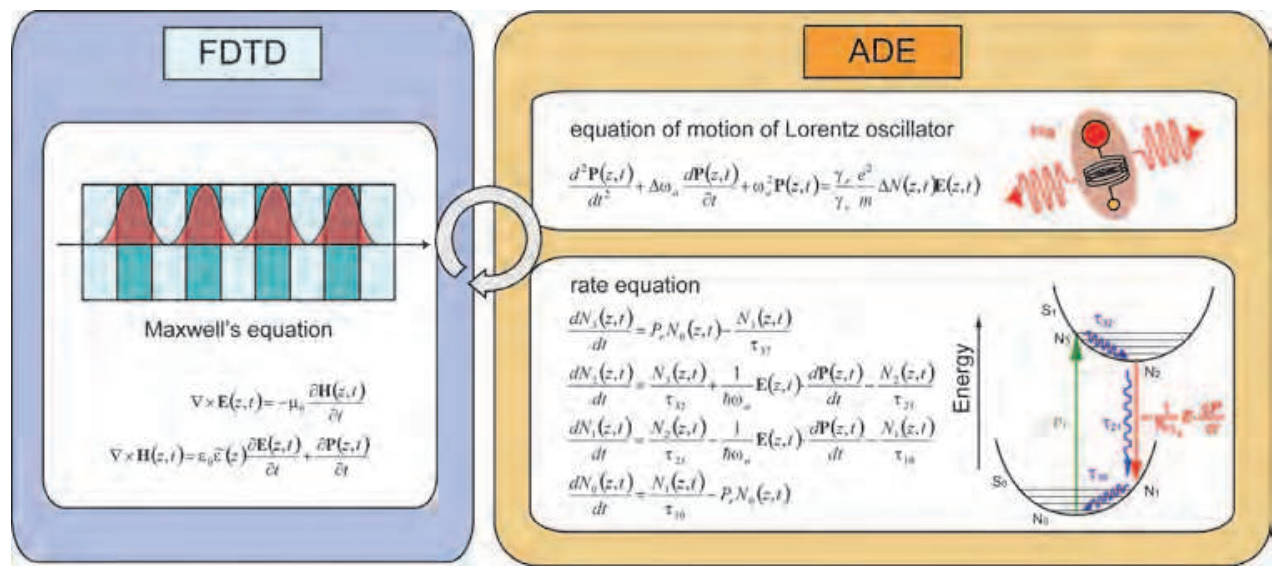

Fig. 4. Schematic representation of ADE-FDTD simulation scheme.

\subsubsection{ADE-FDTD method for the analysis of lasing dynamics in CLCs}

Here numerical procedures for the ADE-FDTD analysis of lasing dynamics will be given. Here we deal with a 1D system, where the time-dependent EM field propagating along the $z$-axis is simulated using Yee's FDTD algorithm (Yee, 1966) to solve the following Maxwell's equations:

$$
\begin{gathered}
\nabla \times \mathbf{E}(z, t)=-\mu_{0} \frac{\partial \mathbf{H}(z, t)}{\partial t} \\
\nabla \times \mathbf{H}(z, t)=\varepsilon_{0} \varepsilon(z) \frac{\partial \mathrm{E}(z, t)}{\partial t}+\frac{\partial \mathbf{P}(z, t)}{\partial t}
\end{gathered}
$$


where $\varepsilon_{0}$ and $\mu_{0}$ are the dielectric permittivity and the magnetic permeability in vacuum, respectively. $\varepsilon(\mathrm{z})$ is dielectric permittivity of the medium and should be tensor for LCs and will be given later. $\mathbf{P}(z, t)$ is the polarization density, which provides a gain mechanism in the laser system. On the basis of the classical electron oscillator (Lorentz) model, one can obtain the following equation of motion of $\mathbf{P}(z, t)$ in the presence of an electric field

$$
\frac{d^{2} \mathbf{P}(z, t)}{d t^{2}}+\Delta \omega_{a} \frac{d \mathbf{P}(z, t)}{\partial t}+\omega_{a}^{2} \mathbf{P}(z, t)=\frac{\gamma_{r}}{\gamma_{c}} \frac{e^{2}}{m} \Delta N(z, t) \mathbf{E}(z, t)
$$

where $\Delta \omega_{\mathrm{a}}=1 / \tau_{21}+2 / T_{2}$ is the full width at half-maximum (FWHM) linewidth of the atomic transition. $T_{2}$ is the mean time between dephasing events and $\omega_{\mathrm{a}}\left(=2 \pi \mathrm{c} / \lambda_{\mathrm{a}}\right)$ is the central frequency of emission. $\Delta N(z, t)\left(=N_{1}(z, t)-N_{2}(z, t)\right)$ is the difference between electron numbers at levels 1 and 2 (Fig. 4), $\gamma_{\mathrm{r}}=1 / \tau_{21}$ and $\gamma_{c}=\left(e^{2} / m\right)\left[\omega_{\mathrm{a}}^{2} /\left(6 \pi \varepsilon_{0} c^{3}\right)\right]$ is the classical rate, $e$ is the electron charge, $m$ is the electron mass and $c$ is the speed of light in vacuum.

The electron numbers at each energy level, $N_{0}(z, t), N_{1}(z, t), N_{2}(z, t)$ and $N_{3}(z, t)$ obey the following rate equations.

$$
\begin{gathered}
\frac{d N_{3}(z, t)}{d t}=P_{r} N_{0}(z, t)-\frac{N_{3}(z, t)}{\tau_{32}} \\
\frac{d N_{2}(z, t)}{d t}=\frac{N_{3}(z, t)}{\tau_{32}}+\frac{1}{\hbar \omega_{a}} \mathbf{E}(z, t) \cdot \frac{d \mathbf{P}(z, t)}{d t}-\frac{N_{2}(z, t)}{\tau_{21}} \\
\frac{d N_{1}(z, t)}{d t}=\frac{N_{2}(z, t)}{\tau_{21}}-\frac{1}{\hbar \omega_{a}} \mathbf{E}(z, t) \cdot \frac{d \mathbf{P}(z, t)}{d t}-\frac{N_{1}(z, t)}{\tau_{10}} \\
\frac{d N_{0}(z, t)}{d t}=\frac{N_{1}(z, t)}{\tau_{10}}-P_{r} N_{0}(z, t)
\end{gathered}
$$

where $\tau_{32}, \tau_{21}$, and $\tau_{10}$, are the lifetimes at each energy levels, and $P_{\mathrm{r}}$ is the pumping rate of electrons from ground state (level 0) to upper energy level (level 3) and is a controlled variable that should be tuned by the pumping intensity in the real experiment. By coupling these equations (1) - (7), numerical simulation of lasing dynamics can be made as schematically shown in Fig. 4. Flow chart of ADE-FDTD algorithm for the analysis of lasing dynamics is summarized in Fig. 5.

In order to deal with anisotropic medium like LC, dielectric permittivity should be represented as tensor. Assuming that LC molecules are uniaxial with optical major axis (director) along $y$-axis and that extraordinary and ordinary refractive indices of LCs are $n_{\mathrm{e}}$ and $n_{\mathrm{o}}$, respectively, then the dielectric tensor of LCs should be represented as

$$
\tilde{\varepsilon}(z)=\left[\begin{array}{ccc}
\varepsilon_{x x}(z) & \varepsilon_{x y}(z) & \varepsilon_{x z}(z) \\
\varepsilon_{y x}(z) & \varepsilon_{y y}(z) & \varepsilon_{y z}(z) \\
\varepsilon_{z x}(z) & \varepsilon_{z y}(z) & \varepsilon_{z z}(z)
\end{array}\right]=\mathbf{R}[-\theta(z)]\left[\begin{array}{ccc}
n_{o}^{2} & 0 & 0 \\
0 & n_{e}^{2} & 0 \\
0 & 0 & n_{o}^{2}
\end{array}\right] \mathbf{R}[\theta(z)]
$$


where $\mathbf{R}[\theta(z)]$ is a rotation matrix about the z-axis and should be expressed as follows,

$$
\mathbf{R}[\theta(z)]=\left[\begin{array}{ccc}
\cos \theta(z) & -\sin \theta(z) & 0 \\
\sin \theta(z) & \cos \theta(z) & 0 \\
0 & 0 & 1
\end{array}\right]
$$

where $\theta(z)$ is the rotated angle from y-axis. By changing $\theta(z)$ gradually as a linear function of $z$, modeling CLC with a helix can be made. Introduction of various types defects as summarized in Fig. 3 can be easily made by modulating this $\theta(z)$ appropriately.

In order to excite the system, a short seed pulse should be launched. $E_{x}$ and $E_{y}$ fields are monitored at a point in the glass until the system reaches a steady state. By Fouriertransforming time domain signals, emission spectra can be analyzed in frequency domain.

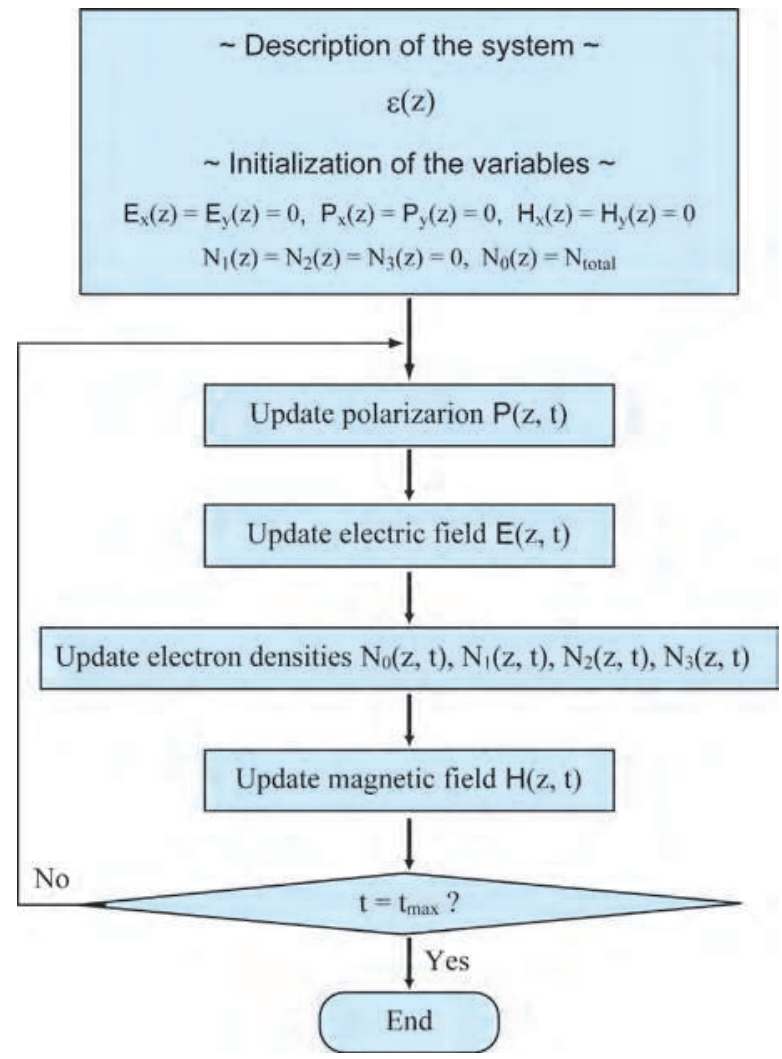

Fig. 5. Flow chart of ADE-FDTD algorithm for the analysis of lasing dynamics.

\subsection{Berreman's $4 \times 4$ matrix for the analysis of transmission and reflection spectra of CLCs}

Berreman's $4 \times 4$ transfer matrix has been widely utilized for the numerical analyses of the optical transmission and reflection spectra in CLC with helical structure (Berreman, 1970). EM fields propagating along the z-axis with frequency $\omega$ are given by 


$$
\frac{d \Psi(z)}{d z}=\frac{i \omega}{c} \mathbf{D}(z) \Psi(z)
$$

where $\Psi(z)=\left[E_{x}(z), H_{y}(z), E_{y}(z), H_{x}(z)\right]^{T}$ and $\mathbf{D}(z)$ is a derivative propagation matrix which should be expressed as follows,

$$
\mathbf{D}(z)=\left[\begin{array}{cccc}
-\frac{\varepsilon_{z x}(z)}{\varepsilon_{z z}(z)} \frac{c k}{\omega} & 1-\frac{1}{\varepsilon_{z z}(z)}\left(\frac{c k}{\omega}\right)^{2} & -\frac{\varepsilon_{z y}(z)}{\varepsilon_{z z}(z)} \frac{c k}{\omega} & 0 \\
\varepsilon_{x x}(z)-\frac{\varepsilon_{x z}(z) \varepsilon_{z x}(z)}{\varepsilon_{z z}(z)} & -\frac{\varepsilon_{x z}(z)}{\varepsilon_{z z}(z)} \frac{c k}{\omega} & \varepsilon_{x y}(z)-\frac{\varepsilon_{x z}(z) \varepsilon_{z y}(z)}{\varepsilon_{z z}(z)} & 0 \\
0 & 0 & 0 & -1 \\
\frac{\varepsilon_{y z}(z) \varepsilon_{z x}(z)}{\varepsilon_{z z}(z)}-\varepsilon_{y x}(z) & -\frac{\varepsilon_{y z}(z)}{\varepsilon_{z z}(z)} \frac{c k}{\omega} & \left(\frac{c k}{\omega}\right)^{2}-\varepsilon_{y y}(z)+\frac{\varepsilon_{y z}(z) \varepsilon_{z y}(z)}{\varepsilon_{z z}(z)} & 0
\end{array}\right]
$$

where $c$ is the speed of light in vacuum, $k$ and $\omega$ are wave number and frequency of light, respectively. $\varepsilon_{\mathrm{ij}}(i, j=x, y$ or $z)$ are dielectric permittivity of LC.

\section{Results and discussion}

In this chapter, our results will be given. In Fig. 6, one of the analyzed CLC laser system with twist defect is schematically represented as an example. A CLC layer is sandwiched between two glass substrates. Physical parameters used in our simulation such as thickness of CLC $t_{\text {CLC }}$ and glass substrates $t_{\mathrm{g}}$, extraordinary and ordinary refractive indices of LCs $n_{\mathrm{e}}$, $n_{\mathrm{o}}$, refractive index of the glass substrate $n_{\mathrm{g}}$, the helical pitch of CLC $p$ are summarized in Table. 1.

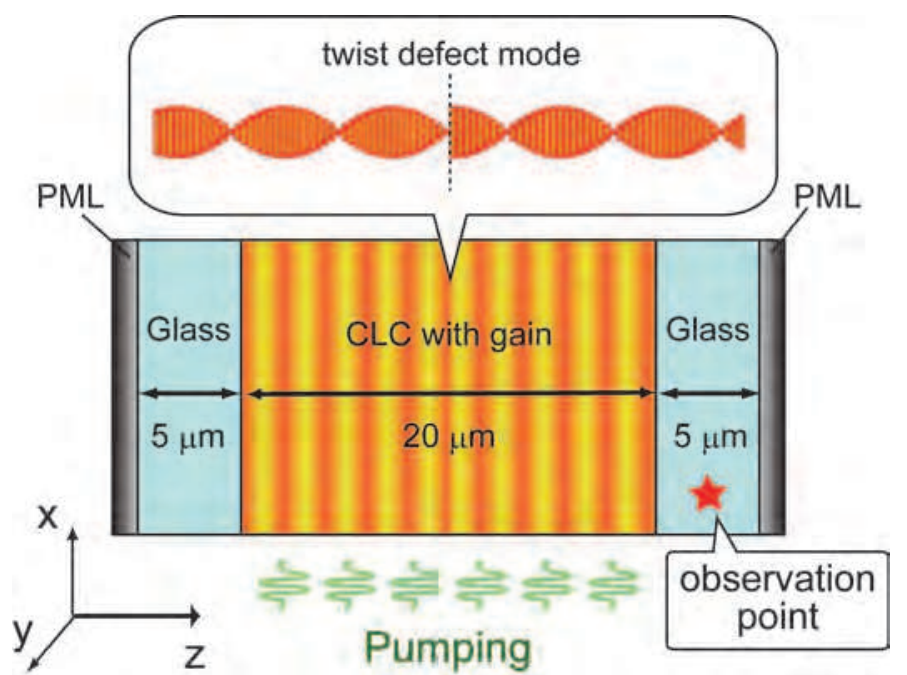

Fig. 6. Schematic representation of CLC laser cavity system with twist-defect at the middle of CLC layer. 
Here we assume that a gain material with a four-level energy structure is introduced in the CLC. The lifetimes at each energy levels, $\tau_{32}, \tau_{21}$, and $\tau_{10}$, are chosen to be similar to those of laser dyes such as coumarine or rhodamine. The total electron density at each point $N_{\text {total }}=$ $N_{0}(z, t)+N_{1}(z, t)+N_{2}(z, t)+N_{3}(z, t)$ should be a constant, and initially, all of them are assumed to be at the ground state, namely, $N_{0}(z, 0)=N_{\text {total }}$ and $N_{1}(z, 0)=N_{2}(z, 0)=N_{3}(z, 0)=$ 0 . These values are also given in Table. 1 .

In order to model an open system, appropriate absorbing boundary conditions should be employed. We have employed perfectly matched layer (PML) (Berenger, 1994). The space increment $\Delta x$ and the time increment $\Delta t$ are chosen to be $10 \mathrm{~nm}, 0.02 \mathrm{fs}$, respectively.

\begin{tabular}{|c|c|}
\hline thickness of CLC: $t_{\text {CLC }}$ & $20 \mu \mathrm{m}$ \\
\hline thickness of glass substrates: $t_{\mathrm{g}}$ & $5 \mu \mathrm{m}$ \\
\hline extraordinary refractive index of LCs: $n_{\mathrm{e}}$ & 1.70 \\
\hline ordinary refractive index of LCs: $n_{\mathrm{o}}$ & 1.50 \\
\hline refractive index of the glass substrate: $n_{\mathrm{g}}$ & 1.50 \\
\hline helical pitch of CLC: $p$ & $400 \mathrm{~nm}$ \\
\hline central wavelength of oscillation of Lorentz oscillator: $\lambda_{\mathrm{a}}$ & $600 \mathrm{~nm}$ \\
\hline lifetime at energy level 3: $\tau_{32}$ & $1.0 \times 10^{-13} \mathrm{~s}$ \\
\hline lifetime at energy level 2: $\tau_{21}$ & $1.0 \times 10^{-9} \mathrm{~s}$ \\
\hline lifetime at energy level 1: $\tau_{10}$ & $1.0 \times 10^{-11} \mathrm{~s}$ \\
\hline total electron density: $N_{\text {total }}$ & $5.5 \times 6.02 \times 10^{23}$ \\
\hline
\end{tabular}

Table 1. Physical parameters of materials and dimensions of device of our model

\subsection{Lasing dynamics}

In Fig. 7, transient responses of electric fields and Fourier-transformed emission spectrum for the case without any defect are summarized. In Fig. 7 (a), transient responses of $E_{x}$ and $E_{y}$ fields monitored at a point in glass are shown. The pumping rate $\left(P_{\mathrm{r}}=1.0 \times 10^{10} \mathrm{~s}^{-1}\right)$ is well above the threshold for the lasing. After a short time ( $3 \mathrm{ps})$, rapid evolution of both $E_{x}$ and $E_{y}$ fields are observed, and after several oscillations, they reach a steady state. In Fig. 7 (b), the steady-state responses of $E_{x}$ and $E_{y}$ fields are shown. A sinusoidal response, which might be due to sharp (monochromatic) lasing emission, is observed. A quarterwavelength phase shift between $E_{x}$ and $E_{y}$ field components can also be recognized. This implies that lasing emission is circularly polarized and this reproduces experimentally observed results well.

Time-windowed time-domain steady-state responses are Fourier-transformed for the evaluation of the power spectrum of the emission. In Fig. 7 (c), the emission spectrum and the transmission spectrum are summarized. A sharp lasing peak appears above the threshold pumping at $600 \mathrm{~nm}$ which corresponds to the higher energy edge of the stop band. In Fig. 7 (d), the emission intensity at the peak wavelength $\left(\lambda_{\mathrm{a}}=600 \mathrm{~nm}\right)$ is shown as a function of pumping rate $P_{\mathrm{r}}$. The threshold pumping rate for lasing can be identified. As discussed above, this can be utilized to pursue the more efficient CLC laser device with reduced lasing threshold. 
(a) $10^{7}$

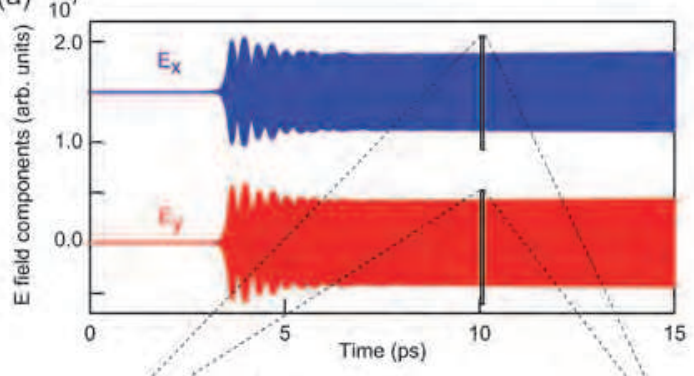

(b) $10^{6} \quad \frac{1}{4} \times \frac{2 \pi}{0_{0}}$

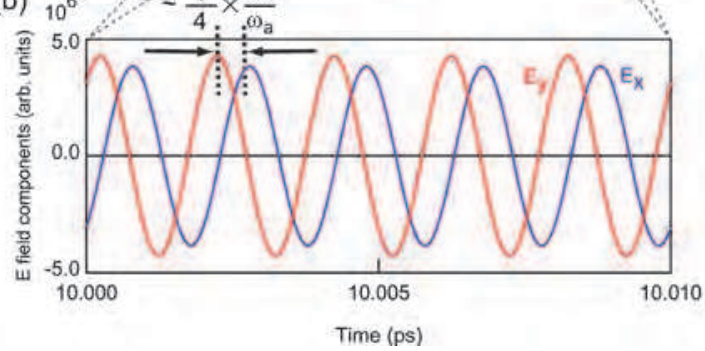

(c)

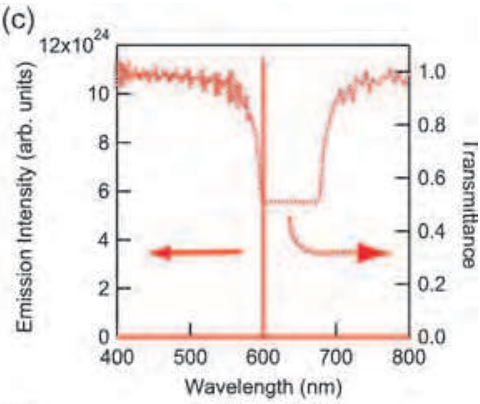

(d)

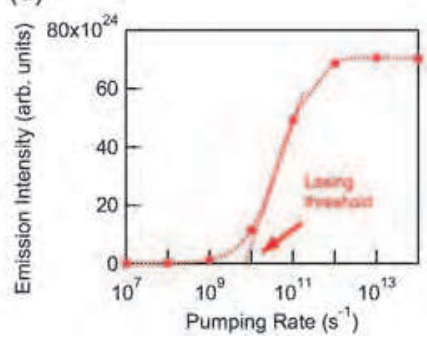

Fig. 7. (a) Transient responses of $E_{\mathrm{x}}$ and $E_{\mathrm{y}}$ field components observed at the glass. Pumping rate $P_{\mathrm{r}}$ is $1.0 \times 10^{10} \mathrm{~s}^{-1}$. (b) Steady-state response at around $10 \mathrm{ps}$. (c) Fourier-transformed emission spectrum and transmittance of CLC with $400 \mathrm{~nm}$ helical pitch. (d) Emission intensity at the lasing peak wavelength $\left(\lambda_{\mathrm{a}}=600 \mathrm{~nm}\right)$ as a function of pumping rate $P_{\mathrm{r}}$.

\subsection{Field distribution}

ADE-FDTD scheme is also suitable to visualize time-dependent spatial distribution of EM fields. This might offer further understanding of underlying physics of CLC lasers. Fig. 8 (a) shows a snapshot of field distribution of $E_{x}$ and $E_{y}$ field components under lasing condition $\left(P_{\mathrm{r}}=1.0 \times 10^{10} \mathrm{~s}^{-1}\right.$ and at $\left.10 \mathrm{ps}\right)$. As can be seen, both $E_{x}$ and $E_{y}$ fields have higher amplitude in the middle of CLC layer, which implies that EM fields are more strongly confined in the middle part of CLC. In our model no gain was introduced in glass, however, both $E_{x}$ and $E_{y}$ fields can be seen and they have almost the same amplitude (envelope) in the whole range in the glass. They might be attributed to the laser emission emitted from CLC laser cavity.

In Fig. 8 (b), magnified snapshots of $E_{x}$ and $E_{y}$ fields in the glass (from 2.0 to $2.6 \mu \mathrm{m}$ ) at different timings around $10 \mathrm{ps}$ are shown. It can be recognized that both $E_{x}$ and $E_{y}$ fields are propagating towards left, and also there is a quarter-wavelength phase shift between $E_{x}$ and $E_{y}$ field oscillations. These facts clearly indicate that a circularly polarized lasing emission is obtained from CLC laser cavity. On the other hand, time-dependent magnified snapshots of $E_{x}$ and $E_{y}$ fields in the CLC (from 15.0 to $15.6 \mu \mathrm{m}$ ) show different characteristics as shown in Fig. 8 (c). Both $E_{x}$ and $E_{y}$ fields do not propagate and they form a standing wave. This also manifests that CLC is working as a distributed feedback laser cavity. There is also a quarterwavelength shift between $E_{x}$ and $E_{y}$ fields, which shows that a standing wave in CLC laser cavity is also circularly polarized and this might explain why circularly polarized lasing is achieved in CLC lasers. In Fig. 8 (d), the angle of oscillation of electric field in the $x-y$ plane 

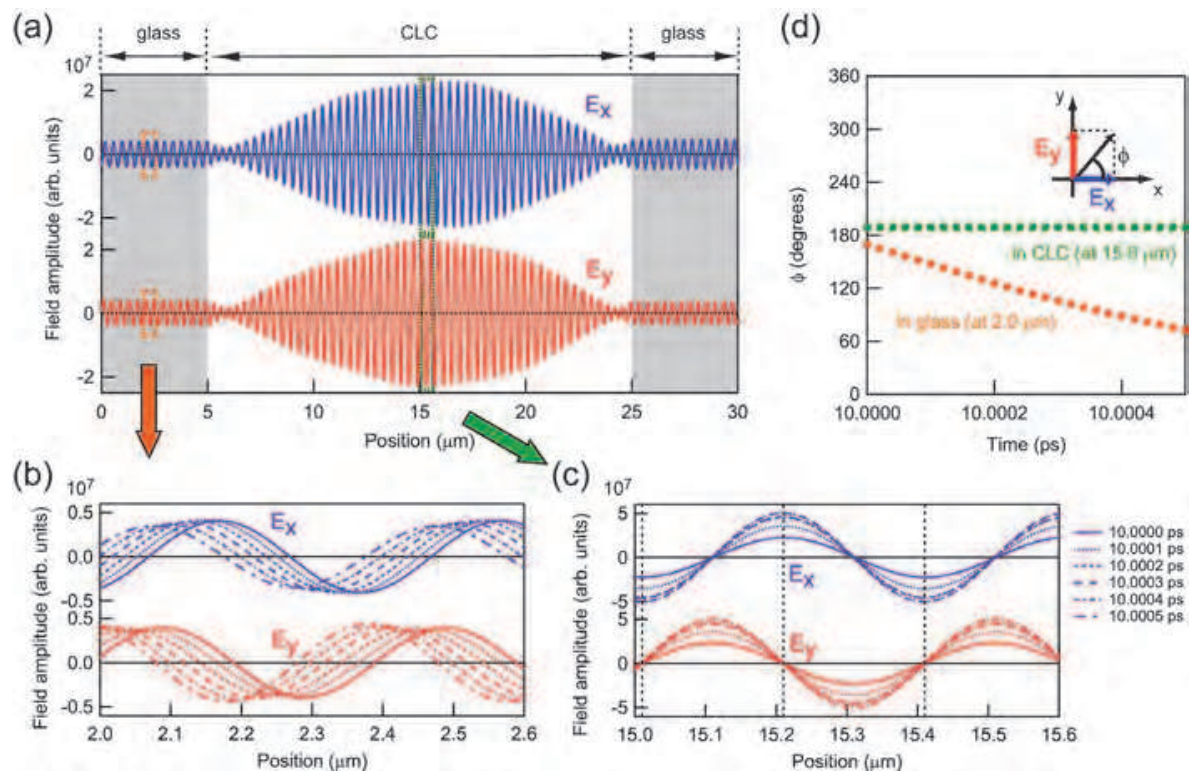

Fig. 8. (a) Spatial distribution of $E_{x}$ and $E_{y}$ field components in CLC and glass at 10 ps. Pumping rate $P_{\mathrm{r}}$ is $1.0 \times 10^{10} \mathrm{~s}^{-1}$. Time-dependent spatial distribution of $E_{x}$ and $E_{y}$ field components in (b) glass and (c) CLC at around 10 ps. (d) the angle of oscillation of electric field in $x-y$ plane $\phi$ as function of time. Inset: the definition of $\phi$.

$\phi(z, t)$ is shown as function of time at a point in glass $(2.0 \mu \mathrm{m})$ and in CLC $(15.0 \mu \mathrm{m})$. The definition of the angle $\phi(z, t)$ is schematically shown in the inset of Fig. 8 (d) and can be deduced as follows.

$$
\phi(z, t)=\arctan \left[\frac{E_{y}(z, t)}{E_{x}(z, t)}\right]
$$

In CLC, the angle $\phi(z=15.0 \mu \mathrm{m}, t)$ does not change at all, which agrees well with the fact that electric field forms a standing wave in CLC. Moreover, it can also be shown that the angle is perpendicular to the director of liquid crystal molecules in the case lasing occurs at the higher edge of the stop band (data is not shown). On the other hand, the angle $\phi(z=2.0$ $\mu \mathrm{m}, t)$ changes linearly with time, which show the angle $\phi$ rotates at a same rate and the value of this rate evaluated from the slope of the plot is roughly equal to the central frequency of lasing emission $\omega_{\mathrm{a}}$. This also manifests that electric fields observed in glass is lasing emission emitted from CLC laser cavity and this is circularly polarized.

\subsection{Twist-defect-mode-lasing}

As discussed above, introduction of various types of defects have been proposed and tested. One of these defects, twist-defect (Fig. 3 (b)), is analyzed here. Most of the conditions for the simulation are kept the same except the thickness of CLC is $10 \mu \mathrm{m}$ here. In Fig. 9 (a) transmission and reflection spectra analyzed by Berreman's $4 \times 4$ transfer matrix are shown. 
(a)

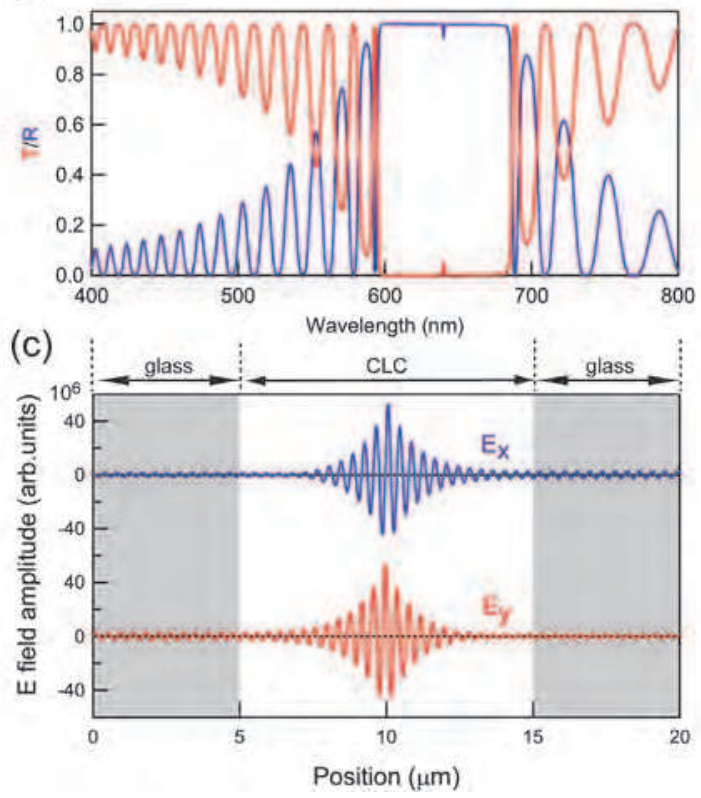

(b)

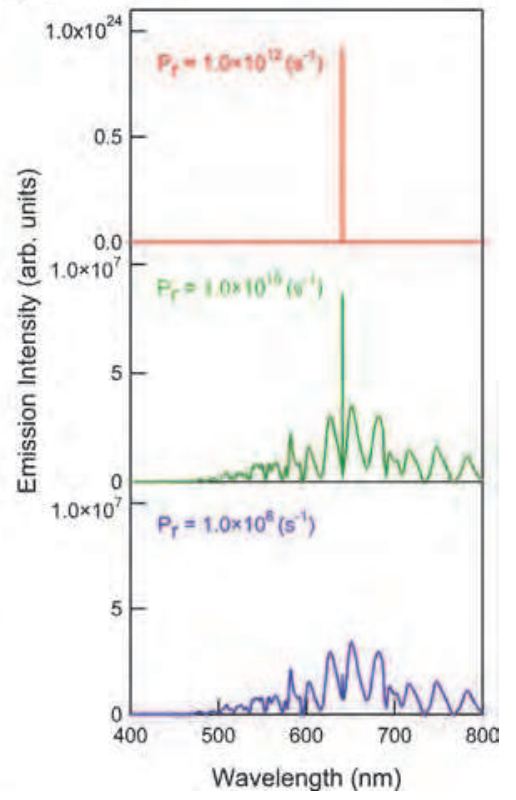

Fig. 9. (a) Transmission and reflection spectra simulated by $4 \times 4$ matrix method. Righthanded circularly polarized light is supposed to normally incident on $10 \mu \mathrm{m}$-thick CLC with $400 \mathrm{~nm}$-pitch right-handed helix and with a twist-defect in the middle. Extraordinary and ordinary refractive indices are 1.70 and 1.50, respectively. (b) Fourier-transformed emission spectra at various pumping rates. Pumping rates $P_{\mathrm{r}}$ are $1.0 \times 10^{8} \mathrm{~s}^{-1}, 1.0 \times 10^{10} \mathrm{~s}^{-1}, 1.0 \times 10^{12} \mathrm{~s}^{-1}$ from bottom to top. (c) Spatial distribution of $E_{x}$ and $E_{y}$ field components in CLC with twistdefect in the middle and glass.

It can be recognized that a sharp transmission/reflection peak appears in the middle of the stop band at around $640 \mathrm{~nm}$. In Fig. 9 (b) Fourier-transformed emission spectra at various pumping rates are summarized. In this case, the central frequency of Lorentz oscillator $\omega_{\mathrm{a}}$ was set to be $2 \pi c /\left(\lambda_{\mathrm{a}}=620 \mathrm{~nm}\right)$ such that band edge lasing $(\sim 600 \mathrm{~nm})$ and twist-defectmode lasing $(\sim 640 \mathrm{~nm})$ can be assumed under similar pumping conditions with similar gain. Above certain threshold pumping around $P_{\mathrm{r}}=1.0 \times 10^{10} \mathrm{~s}^{-1}$, sharp lasing peak appears at around $640 \mathrm{~nm}$, which corresponds to the energy of introduced twist-defect state. When pumping rate is increased further, another lasing peak appears at around $600 \mathrm{~nm}$ which corresponds to higher energy edge of the stop band (data is not shown). These results imply that lasing at twist-defect-mode can be obtained with lowered threshold than that at the edge of the stopband and also shows that the introduction of defect is quite effective for the reduction of lasing threshold.

Fig. 9 (c) shows a snapshot of field distribution of $E_{x}$ and $E_{y}$ field components under lasing condition. Field distribution (envelope) in CLC with twist-defect is quite different from that without a defect (Fig. 8 (a)). Electric fields are strongly localized at the site where the twist- 
defect is introduced. This strong confinement of EM fields might enable strong light-matter interaction and result in lower lasing threshold.

\section{Conclusions}

In conclusion, we numerically investigated the lasing dynamics in CLC as a 1D chiral PBG material by the ADE-FDTD approach which couples FDTD with ADEs such as the rate equation in a four-level energy structure and the equation of motion of Lorentz oscillator. This technique enables us to analyze lasing dynamics from various viewpoints such as timedependent emission dynamics, Fourier-transformed emission spectra and time-dependent field distributions. Band edge circularly polarized lasing was successfully reproduced above threshold pumping. Through the analysis of time-dependent EM field distributions, it is shown that circularly polarized lasing emission is obtained from CLC laser cavity. It is also shown that standing wave with quarter-wavelength phase shift between orthogonal field components is obtained in CLC, which might explain CLC works as distributed feedback laser cavity for circularly polarized lasing emission. With the introduction of twist-defect, lasing emission at defect-mode energy with lower lasing threshold was obtained. It is also shown that the field distribution in CLC with twist-defect is introduced is quite different from that without any defect. ADE-FDTD approach might be utilized to find more efficient device architecture for obtaining a lower lasing threshold.

\section{Acknowledgment}

The presented works have been done with one of former graduate students of our research group, Mr. Masahiro Kitaguchi.

\section{References}

Berenger, J.-P. (1994). A perfectly matched layer for the absorption of electromagnetic waves. Journal of Computational Physics, Vol.114, No.2, pp.185-200.

Bermel, P., Lidorikis, E., Fink, Y. \& Joannopoulos, J. D. (2006). Active materials embedded in photonic crystals and coupled to electromagnetic radiation. Physical Review B, Vol.73, No.16, pp.165125-1-8.

Berreman, D. W. \& Scheffer, T. J. (1970). Bragg reflection of light from single-domain cholesteric liquid-crystal films. Physical Review Letters, Vol.25, No.9, pp.577581.

Chang, S.-H. \& Taflove, A. (2004). Finite-difference time-domain model of lasing action in a four-level two-electron atomic system. Optics Express, Vol.12, No.16, pp.38273833.

Coles, H. \& Morris, S. (2010). Liquid-crystal lasers. Nature Photonics, Vol.4, No.10, pp.676685.

de Gennes, P. G. \& Prost, J. (1995). The Physics of Liquid Crystals (2nd ed.), Oxford University Press, ISBN 9780198517856, New York.

Dowling, J. P., Scalora, M., Bloemer. M. J., Bowden, C. M. (1994). The Photonic Band-edge Laser - A New Approach to Gain Enhancement. Journal of Applied Physics, Vol.75, No.4, pp.1896-1899. 
Jiang, X. \& Soukoulis, C. M. (2000). Time Dependent Theory for Random Lasers. Physical Review Letters, Vol.85, No.1, pp.70-73.

Joannopoulos, J. D., Meade, R. D. \& Winn, J. N. (1995). Photonic Crystals: Molding the Flow of Light, Princeton University Press, ISBN 0691037442, New Jersey

John, S. (1987). Strong localization of photons in certain disordered dielectric superlattices. Physical Review Letters, Vol.58, No.23, pp.2486-2489.

Kopp, V. I., Fan, B., Vithana, H. K. \& Genack, A. Z. (1998). Low-threshold lasing at the edge of a photonic stop band in cholesteric liquid crystals. Optics Letters, Vol.23, No.21, pp.1707-1709.

Kopp, V. I. \& Genack, A. Z. (2002). Twist defect in chiral photonic structures. Physical Review Letters, Vol.89, No.3, pp.033901-1-4.

Matsui, T., Ozaki, M. \& Yoshino, K. (2004). Tunable photonic defect modes in a cholesteric liquid crystal induced by optical deformation of helix. Physical Review E, Vol.69, No.6, pp.061715-1-4.

Matsui, T. \& Kitaguchi, M. (2010). Finite-Difference Time-Domain Analysis of Laser Action in Cholesteric Photonic Liquid Crystal. Applied Physics Express, Vol.3, No.6, pp.061701-1-3.

Nagra, A. S. \& York, R. A. (1998). FDTD Analysis of Wave Propagation in Nonlinear Absorbing and Gain Media. IEEE Transactions on Antennas and Propagation, Vol.46, No.3, pp.334-340.

Ozaki, M., Ozaki, R., Matsui, T. \& Yoshino, K. (2003). Twist-Defect-Mode Lasing in Photopolymerized Cholesteric Liquid Crystal. Japanese Journal of Applied Physics, Vol.42, No.5A, pp.L472-L475.

Ozaki, R., Sanda, T., Yoshida, H., Matsuhisa, Y., Ozaki, M. \& Yoshino, K. (2006). Defect Mode in Cholesteric Liquid Crystal Consisting of Two Helicoidal Periodicities. Japanese Journal of Applied Physics, Vol.45, No.1B, pp.493-496.

Redding, B., Shi, S., Creazzo, T. \& Prather, D. W. (2008). Electromagnetic modeling of active silicon nanocrystal waveguides. Optics Express, Vol.16, No.12, pp. 87928799 .

Schmidtke, J., Stille, W. \& Finkelmann, H. (2003). Defect mode emission of a dye doped cholesteric polymer network. Physical Review Letters, Vol.90, No.8, pp.083902$1-4$.

Shi, S. \& Prather, D. W. (2007). Lasing dynamics of a silicon photonic crystal microcavity. Optics Express, Vol.15, No.16, pp.10294-10302.

Takanishi, Y., Tomoe, N., Ha, N. Y., Toyooka, T., Nishimura, S., Ishikawa, K. \& Takezoe, H. (2007). Defect-mode lasing from a three-layered helical cholesteric liquid crystal structure. Japanese Journal of Applied Physics, Vol.46, No.6A, pp.3510-3513.

Taflove, A., \& Hagness, S. C. (2005). Computational Electrodynamics: The Finite-Difference TimeDomain Method (3rd ed.), Artech House, ISBN 1580538320, Norwood, MA.

Vanneste, C. \& Sebbah, P. (2001). Selective Excitation of Localized Modes in Active Random Media. Physical Review Letters, Vol.87, No.18, pp.183903-1-4.

Yablonovitch, E. (1987). Inhibited spontaneous emission in solid-state physics and electronics. Physical Review Letters, Vol.58, No.20, pp.2059-2062.

Yang, Y.-C., Kee, C.-S., Kim, J.-E., Park., H.-Y., Lee. J.-C. \& Jeon., Y.-J. (1999). Photonic defect modes of cholesteric liquid crystals. Physical Review E, Vol.60, No.6, pp.68526854 . 
Yee, K. S. (1966). Numerical solution of initial boundary value problems involving maxwell's equations in isotropic media. IEEE Transactions on Antennas and Propagation, Vol.14, No.3, pp.302-307. 


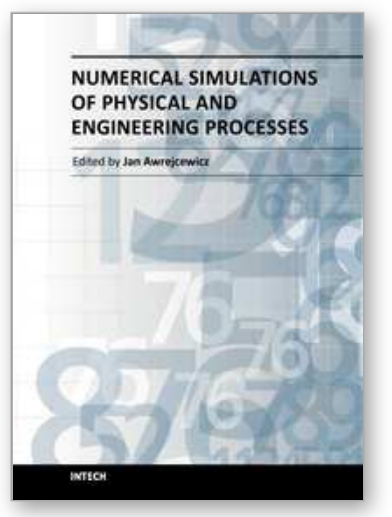

\author{
Numerical Simulations of Physical and Engineering Processes \\ Edited by Prof. Jan Awrejcewicz
}

ISBN 978-953-307-620-1

Hard cover, 594 pages

Publisher InTech

Published online 26, September, 2011

Published in print edition September, 2011

Numerical Simulations of Physical and Engineering Process is an edited book divided into two parts. Part I devoted to Physical Processes contains 14 chapters, whereas Part II titled Engineering Processes has 13 contributions. The book handles the recent research devoted to numerical simulations of physical and engineering systems. It can be treated as a bridge linking various numerical approaches of two closely interrelated branches of science, i.e. physics and engineering. Since the numerical simulations play a key role in both theoretical and application oriented research, professional reference books are highly needed by pure research scientists, applied mathematicians, engineers as well post-graduate students. In other words, it is expected that the book will serve as an effective tool in training the mentioned groups of researchers and beyond.

\title{
How to reference
}

In order to correctly reference this scholarly work, feel free to copy and paste the following:

Tatsunosuke Matsui (2011). Numerical Simulation of Lasing Dynamics in Choresteric Liquid Crystal Based on ADE-FDTD Method, Numerical Simulations of Physical and Engineering Processes, Prof. Jan Awrejcewicz (Ed.), ISBN: 978-953-307-620-1, InTech, Available from: http://www.intechopen.com/books/numericalsimulations-of-physical-and-engineering-processes/numerical-simulation-of-lasing-dynamics-in-chorestericliquid-crystal-based-on-ade-fdtd-method

\section{INTECH}

open science | open minds

\author{
InTech Europe \\ University Campus STeP Ri \\ Slavka Krautzeka 83/A \\ 51000 Rijeka, Croatia \\ Phone: +385 (51) 770447 \\ Fax: +385 (51) 686166 \\ www.intechopen.com
}

\author{
InTech China \\ Unit 405, Office Block, Hotel Equatorial Shanghai \\ No.65, Yan An Road (West), Shanghai, 200040, China \\ 中国上海市延安西路65号上海国际贵都大饭店办公楼 405 单元 \\ Phone: +86-21-62489820 \\ Fax: $+86-21-62489821$
}


(C) 2011 The Author(s). Licensee IntechOpen. This chapter is distributed under the terms of the Creative Commons Attribution-NonCommercialShareAlike-3.0 License, which permits use, distribution and reproduction for non-commercial purposes, provided the original is properly cited and derivative works building on this content are distributed under the same license. 